\title{
Postoperative imaging for detection of recurrent arteriovenous malformations in children
}

\author{
Peter F. Morgenstern, MD, ${ }^{1}$ Caitlin E. Hoffman, MD, ${ }^{1}$ Gary Kocharian, BA, ${ }^{2}$ Ranjodh Singh, BPhil, ${ }^{2}$ \\ Philip E. Stieg, PhD, MD, ${ }^{1,2}$ and Mark M. Souweidane, MD ${ }^{1,2}$

\begin{abstract}
'Department of Neurological Surgery, NewYork-Presbyterian Hospital, Weill Cornell Medical Center; and 2Weill Cornell Medical
\end{abstract} \\ College, New York, New York
}

OBJECTIVE The optimal method for detecting recurrent arteriovenous malformations (AVMs) in children is unknown. An inherent preference exists for MR angiography (MRA) surveillance rather than arteriography. The validity of this strategy is uncertain.

METHODS A retrospective chart review was performed on pediatric patients treated for cerebral AVMs at a single institution from 1998 to 2012. Patients with complete obliteration of the AVM nidus after treatment and more than 12 months of follow-up were included in the analysis. Data collection focused on recurrence rates, associated risk factors, and surveillance methods.

RESULTS A total of 45 patients with a mean age of 11.7 years (range 0.5-18 years) were treated for AVMs via surgical, endovascular, radiosurgical, or combined approaches. Total AVM obliteration on posttreatment digital subtraction angiography (DSA) was confirmed in 27 patients, of whom the 20 with more than 12 months of follow-up were included in subsequent analysis. The mean follow-up duration in this cohort was 5.75 years (median 5.53 years, range 1.11-10.64 years). Recurrence occurred in 3 of 20 patients (15\%). Two recurrences were detected by surveillance DSA and 1 at the time of rehemorrhage. No recurrences were detected by MRA. Median time to recurrence was 33.6 months (range 19-71 months). Two patients (10\%) underwent follow-up DSA, 5 (25\%) had DSA and MRI/MRA, 9 (45\%) had MRI/MRA only, $1(5 \%)$ had CT angiography only, and $3(15 \%)$ had no imaging within the first 3 years of follow-up. After 5 years posttreatment, 2 patients (10\%) were followed with MRI/MRA only, $2(10 \%)$ with DSA only, and $10(50 \%)$ with continued DSA and MRI/MRA.

CONCLUSIONS AVM recurrence in children occurred at a median of 33.6 months, when MRA was more commonly used for surveillance, but failed to detect any recurrences. A recurrence rate of $15 \%$ may be an underestimate given the reliance on surveillance MRA over angiography. A new surveillance strategy is proposed, taking into account exposure to diagnostic radiation and the potential for catastrophic rehemorrhage.

http://thejns.org/doi/abs/10.3171/2015.6.PEDS14708

KEY WORDS arteriovenous malformation; cerebral angiography; magnetic resonance imaging; recurrence; vascular disorders

$\mathrm{A}$ RTERIOVENOUS malformation (AVM) is the most common cause of intracerebral hemorrhage (ICH) in children. ${ }^{4,5}$ The annual risk of hemorrhage in pediatric AVM is approximately $3.2 \%$ per year, ${ }^{17}$ with $5 \%-10 \%$ risk of death and up to $50 \%$ risk of neurological morbidity. ${ }^{1,12,15,17}$ Early and complete treatment of these malformations in children is therefore important. Current treatment options include microsurgery, endovascular embolization, radiosurgery, or combined therapy.
Even with confirmed complete obliteration of the AVM nidus, recurrence is reported in $5.5 \%-14.3 \%$ of children, while AVM recurrence has infrequently been reported in adults. ${ }^{1,2,9,10,16,23,29}$ Although the underlying cause of this apparently different biology is unclear, ${ }^{26}$ it is an important factor to consider in the management and postoperative follow-up of pediatric patients with AVMs. In addition to patient age, deep venous drainage and diffuse nidus have been identified as potential risk factors for recurrence. ${ }^{14,16,18}$

ABBREVIATIONS $\mathrm{AChA}=$ anterior choroidal artery; $\mathrm{AVM}=$ arteriovenous malformation; $\mathrm{CTA}=\mathrm{CT}$ angiography; $\mathrm{DSA}=$ digital subtraction angiography; $\mathrm{EVD}=$ external ventricular drain; ICA = internal carotid artery; ICH = intracerebral hemorrhage; MRA = MR angiography; $m R S=$ modified Rankin Scale.

SUBMITTED January 20, 2015. ACCEPTED June 30, 2015.

INCLUDE WHEN CITING Published online October 30, 2015; DOI: 10.3171/2015.6.PEDS14708. 
Despite the need for accurate and timely diagnosis of recurrence, the optimal follow-up strategy in children has not been demonstrated. Some studies recommend imaging 6-12 months after a negative postoperative digital subtraction angiogram to rule out recurrent or residual AVM..$^{13,16,20,29}$ With reported cases of recurrence occurring 3 months to 16 years after primary treatment, $, 1,2,13,16,26,29$ a more extended imaging protocol is likely necessary to capture recurrence and prevent associated morbidity. Follow-up imaging, however, carries additional risk, cost, and time. The appropriate follow-up modality and timing are therefore subjects of debate, particularly in light of concerns regarding radiation exposure and general anesthesia in children. ${ }^{7,22,30}$ We present our experience with surveillance for AVM recurrence, associated risk factors, and clinical outcome, while considering the implications of these findings for follow-up protocols after treatment.

\section{Methods}

This retrospective study was approved by the Weill Cornell Medical College Institutional Review Board. Patients younger than 18 years of age diagnosed with AVM by digital subtraction angiography (DSA) from 1998 to 2012 at the primary institution were identified, and those with complete nidus obliteration after treatment with more than 12 months of follow-up were included in the final analysis. All available inpatient and outpatient clinical records were reviewed. Data relevant to diagnostic studies, treatment modalities, follow-up, recurrence, and clinical outcome measures were collected. Spetzler-Martin grade was calculated based on radiological reports of nidus size, drainage, and location. Modified Rankin Scale (mRS) scores were determined from provider documentation at presentation and at each follow-up encounter. The followup protocol at this institution included an intraoperative or immediately postoperative angiogram, followed by annual MRI and angiography at 5 and 10 years. Patient follow-up in this cohort in some cases varied due to other unforeseen issues related to scheduling, insurance status, and decisions made by providers other than the primary surgeon. For these reasons, patients were followed with a combination of CT angiography (CTA), MRI, and in some cases, MR angiography (MRI/MRA) and/or DSA at varying time points.

\section{Results}

Twenty-four girls and 21 boys with a mean age of 11.7 years (range 0.5-18 years) were diagnosed with AVMs during the study period. The most common presenting finding was intracranial hemorrhage $(66.7 \%)$. The most common presenting symptoms were headache (55.6\%) and weakness (28.9\%). Supratentorial lesions comprised $97.8 \%$ of the group. Patients were treated with microsurgical resection (15.6\%), embolization alone (15.6\%), radiosurgery alone (4.4\%), embolization and microsurgical resection (42.2\%), or embolization and radiosurgery (4.4\%). Seven patients $(15.6 \%)$ received no treatment, while one patient's treatment status was unavailable (2.2\%; Table 1). Posttreatment DSA confirmed total obliteration of the nidus in 27 of the 45 patients, of whom 20 had more than 1 year of follow- up. This subset of 20 patients was used for all subsequent analysis. The mean follow-up duration was 5.75 years (median 5.53 years, range 1.11-10.64 years). The characteristics of this group were similar to those of the overall cohort with the exception of treatment strategy, which included a higher percentage of patients undergoing craniotomy. Spetzler-Martin Grade II and III lesions comprised 64.5\% of the entire cohort, and $70 \%$ of the completely treated patients $(p=0.37$, Table 1$)$. Patients in the completely treated group had a higher preoperative mRS score than those excluded from the study. On follow-up assessment, however, there was no difference between the groups (Table 2).

Surveillance for recurrence in patients with complete nidus obliteration after treatment consisted of CTA, MRI/ MRA, DSA, or a combination of these imaging modalities as dictated by the provider and described above. In addition to the intraoperative or immediate postoperative imaging study confirming no residual lesion, 2 patients $(10 \%)$ had follow-up DSA, 5 (25\%) had DSA and MRI/ MRA, 9 (45\%) had MRI/MRA only, 1 (5\%) had CTA only, and $3(15 \%)$ had no imaging within the first 3 years of follow-up. At 5 years posttreatment, 2 patients (10\%) were followed with MRI/MRA only, 2 (10\%) with DSA only, and 10 (50\%) with continued DSA and MRI/MRA (Table 3). Of the follow-up MRI/MRA studies, 6 were Gdenhanced MRI only, while the remaining 7 were a combination of Gd-enhanced MRI and unenhanced 3D timeof-flight MRA.

Recurrence was observed in $3(15 \%)$ of 20 patients aged 7-18 years (Fig. 1). The median time to recurrence was 33.6 months (mean 41.5 months, range 19-71 months). Recurrences were identified in 2 cases by surveillance DSA, and in the third following rehemorrhage (Table 4). Two recurrences were identified after previously normal MRI/MRA (interval of 6 months and 2 years, respectively). One of the 3 patients with recurrence had a negative digital subtraction angiogram more than 1 year postoperatively, 2 years prior to the study demonstrating recurrence. No other associated risk factors for recurrence-including emergent treatment $(\mathrm{p}=0.52)$, pretreatment hemorrhage $(\mathrm{p}=0.99)$, Spetzler-Martin grade $(\mathrm{p}=0.99)$, AVM size $(\mathrm{p}$ $=0.99)$ and drainage $(\mathrm{p}=0.54)$, and associated aneurysms $(p=0.99)$-were found to correlate with recurrence in this study, and no recurrences were diagnosed by other imaging modalities.

\section{Illustrative Cases of Recurrent AVMs Case 1}

This 6-year-old girl presented with headache, vomiting, and seizure, at which time a Spetzler-Martin Grade II right parietal AVM with posterior middle cerebral artery feeders and a draining vein into the transverse sinus was diagnosed using DSA. She required external ventricular drain (EVD) placement and underwent embolization followed by craniotomy for resection. Intraoperative DSA demonstrated no residual AVM filling. She recovered well and underwent DSA 18 months later, revealing a recurrent AVM in the right lateral ventricle with feeders from the right anterior choroidal artery (AChA). The family declined treatment, and she continues under observation. 
TABLE 1. Characteristics of 45 patients with AVMs, included and excluded patients

\begin{tabular}{|c|c|c|c|c|}
\hline Characteristic & Overall Cohort & Included Patients & Excluded Patients & $p$ Value* \\
\hline No. of patients & 45 & 20 & 25 & \\
\hline $\operatorname{Sex}(\%)$ & & & & 0.69 \\
\hline $\mathrm{M}$ & $21(46.7)$ & $10(50)$ & $11(44)$ & \\
\hline $\mathrm{F}$ & $24(53.3)$ & $10(50)$ & $14(56)$ & \\
\hline \multicolumn{5}{|l|}{ Age (yrs) } \\
\hline Mean & 11.7 & 11.2 & 12.06 & \\
\hline Median & 13 & 11 & 14 & \\
\hline Range & $0.5-18$ & $3-18$ & $0.5-17$ & \\
\hline Presenting signs \& symptoms (\%) & & & & 0.84 \\
\hline Hemorrhage & $30(66.7)$ & $17(85)$ & $13(52)$ & \\
\hline Headache & $25(55.6)$ & $11(55)$ & $14(56)$ & \\
\hline Hemiparesis & $13(28.9)$ & $8(40)$ & $5(20)$ & \\
\hline Seizure & $12(26.7)$ & $6(30)$ & $6(24)$ & \\
\hline Altered mental status & $11(24.4)$ & $6(30)$ & $5(20)$ & \\
\hline Visual deficit & $4(8.9)$ & $2(10)$ & $2(8)$ & \\
\hline Cranial nerve deficit & $2(4.4)$ & $1(5)$ & $1(4)$ & \\
\hline Asymptomatic & $3(6.7)$ & $1(5)$ & $2(8)$ & \\
\hline Spetzler-Martin Grade (\%) & & & & 0.37 \\
\hline I & $6(13.3)$ & $4(20)$ & $2(8)$ & \\
\hline$\|$ & $13(28.9)$ & $7(35)$ & $6(24)$ & \\
\hline III & $16(35.6)$ & $7(35)$ & $9(36)$ & \\
\hline IV & $9(20)$ & $2(10)$ & $7(28)$ & \\
\hline V & $1(2.2)$ & $0(0)$ & $1(4)$ & \\
\hline AVM location (\%) & & & & 0.96 \\
\hline Frontal & $14(31.1)$ & $6(30)$ & $8(32)$ & \\
\hline Temporal & $10(22.2)$ & $4(20)$ & $6(24)$ & \\
\hline Parietal & $7(15.6)$ & $4(20)$ & $3(12)$ & \\
\hline Occipital & $5(11.1)$ & $2(10)$ & $3(12)$ & \\
\hline Thalamus/basal ganglia & $7(15.6)$ & $3(15)$ & $4(16)$ & \\
\hline Brainstem & $1(2.2)$ & $1(5)^{\prime}$ & $0(0)$ & \\
\hline Intraventricular & $1(2.2)$ & $0(0)$ & $1(4)$ & \\
\hline Treatment strategy (\%) & & & & 0.02 \\
\hline Endovascular & $7(15.6)$ & $1(5)$ & $6(24)$ & \\
\hline Craniotomy & $7(15.6)$ & $4(20)$ & $3(12)$ & \\
\hline Radiosurgery & $2(4.4)$ & $0(0)$ & $2(8)$ & \\
\hline Endovascular + craniotomy & $19(42.2)$ & $13(65)$ & $6(24)$ & \\
\hline Endovascular + radiosurgery & $2(4.4)$ & $2(10)$ & $0(0)$ & \\
\hline Observation & $7(15.6)$ & $0(0)$ & $7(28)$ & \\
\hline Unavailable & $1(2.2)$ & $0(0)$ & $1(4)$ & \\
\hline
\end{tabular}

* $p$ value from chi-square test or Fisher's exact test, as appropriate.

TABLE 2. Modified Rankin Scale scores in the overall cohort compared with a subset of patients with complete AVM obliteration after treatment

\begin{tabular}{|c|c|c|c|c|c|c|}
\hline \multirow[b]{2}{*}{ Score } & \multicolumn{2}{|c|}{ Overall Cohort $(n=40)$} & \multicolumn{2}{|c|}{ Excluded Patients $(n=21)$} & \multicolumn{2}{|c|}{ Complete Treatment $(n=19)$} \\
\hline & Presentation & Follow-Up & Presentation & Follow-Up & Presentation & Follow-Up \\
\hline 0 & 1 & 12 & 0 & 6 & 1 & 6 \\
\hline 1 & 21 & 17 & 13 & 10 & 8 & 7 \\
\hline 2 & 3 & 7 & 3 & 3 & 0 & 5 \\
\hline 3 & 1 & 3 & 1 & 1 & 0 & 1 \\
\hline 4 & 8 & 1 & 3 & 1 & 5 & 0 \\
\hline 5 & 6 & 0 & 1 & 0 & 5 & 0 \\
\hline Mean & 2.3 & 1.1 & 1.9 & 1.1 & 2.8 & 1.1 \\
\hline
\end{tabular}


TABLE 3: Follow-up modality in 20 patients with AVMs

\begin{tabular}{lccc}
\hline & \multicolumn{3}{c}{ Follow-Up Duration } \\
\cline { 2 - 4 } Follow-Up Modality & $<12$ Mos (\%) & $1-3$ Yrs $(\%)$ & $>5$ Yrs $(\%)$ \\
\hline DSA & $2(10)$ & $2(10)$ & $2(10)$ \\
MRI/MRA & $3(15)$ & $11(55)$ & $2(10)$ \\
CTA & $1(5)$ & $1(5)$ & $1(5)$ \\
DSA \& MRI/MRA & $2(10)$ & $3(15)$ & $10(50)$ \\
No Imaging & $12(60)$ & $3(15)$ & $5(25)$ \\
\hline
\end{tabular}

\section{Case 2}

A 14-year-old girl presented with right hemiparesis and altered mental status and was found to have an ICH requiring placement of an EVD. She underwent DSA, which demonstrated a Spetzler-Martin Grade IV left thalamic AVM. The AVM was supplied by the left AChA, posterior choroidal, and posterior cerebral artery feeders, and drained into the left internal cerebral vein. She underwent $50 \%$ embolization followed by stereotactic radiosurgery. Follow-up MRI/MRA was unrevealing at 18 months, and DSA at 20 months demonstrated resolution of the AVM. Follow-up MRI/MRA 3.5 years after treatment was stable, and she presented with a new left thalamic hemorrhage 6 months later. Angiography at that time revealed a recurrent AVM in the left thalamus, which was treated with stereotactic radiosurgery.

\section{Case 3}

An 8-year-old girl presented with left hemiplegia and underwent emergency evacuation of a right frontoparietal ICH. Subsequent DSA demonstrated a Spetzler-Martin Grade II AVM with right pericallosal artery feeders and deep drainage. She underwent preoperative embolization followed by craniotomy and resection of the AVM. Postoperative DSA showed no residual filling, and MRI/MRA was completed annually at 2,3, and 4 years. At 6 years, DSA demonstrated a small local recurrence. This nidus was embolized and completely resected without complication.

\section{Discussion}

Arteriovenous malformations of the brain may develop as early as the third week of gestation but typically remain asymptomatic until late adolescence or early adulthood. ${ }^{2,8,19}$ Only $18 \%-20 \%$ of patients with AVMs present before 15 years of age, ${ }^{13,17}$ with hemorrhage and seizure being the most common primary events. ${ }^{1,5,31,32}$ Treatment of AVMs includes microsurgical resection, radiosurgery, endovascular embolization, or a combination of these strategies. $3,6,24,28$

Accumulating evidence has demonstrated that AVMs recur in children..$^{1,2,14,16}$ The rate of hemorrhage associated with recurrent AVMs is not well defined, largely due to relatively small numbers of patients available for study. We confirm in our series that recurrences do occur in the pediatric population, and demonstrate that they can present with hemorrhage despite recent negative imaging. This raises an important question: what is the best modality for surveillance of these children?
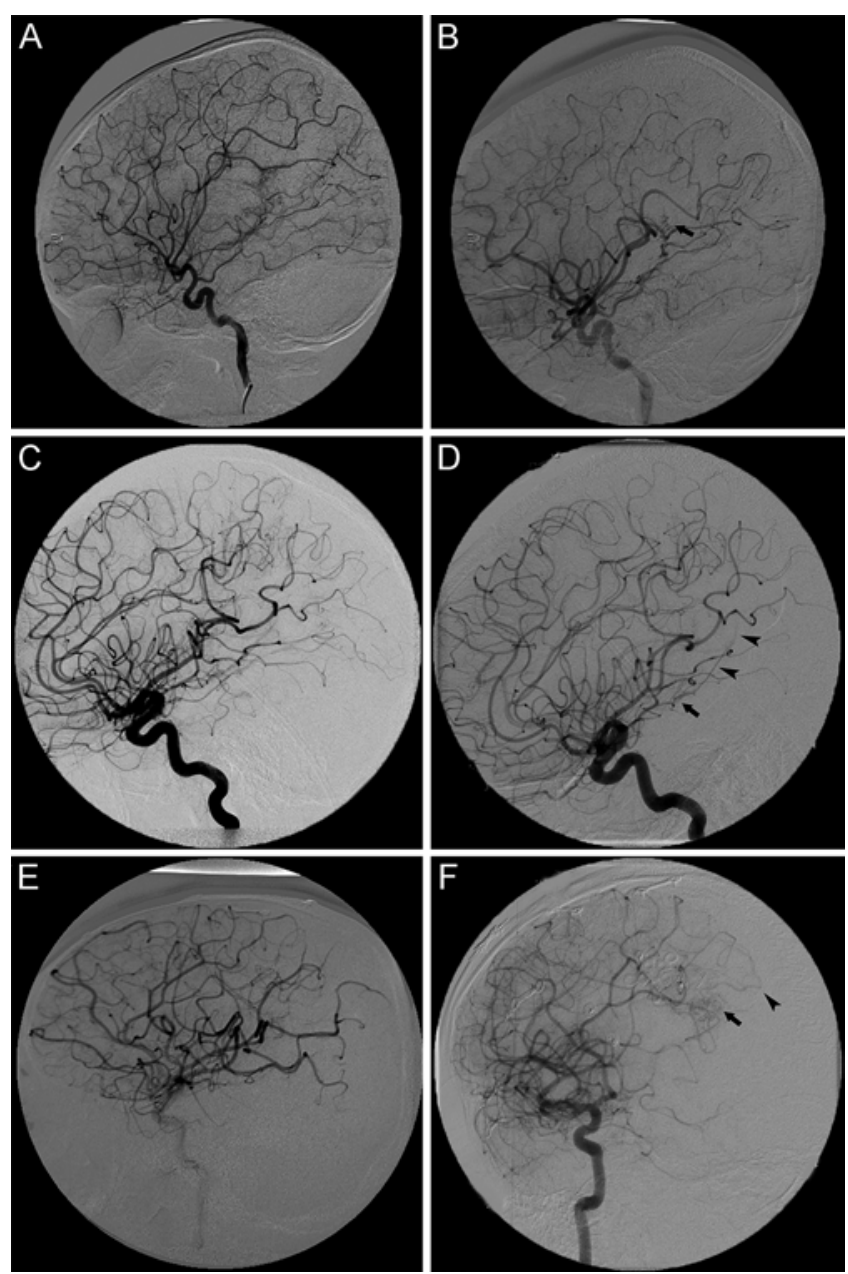

FIG. 1. Angiographic images demonstrating recurrent AVMs in the 3 cases. Case 1. A: Lateral projection, right internal carotid artery (ICA) injection shows no evidence of residual AVM after embolization and microsurgical resection. B: An abnormal network of vessels is seen filling via the right AChA (arrow). Case 2. C: Image shows no evidence of residual nidus on lateral projection of a left ICA injection after embolization and radiosurgery for a left thalamic AVM. D: Angiogram at recurrence demonstrates abnormal arteries in the left thalamus and mesial temporal lobe (arrow) supplied by the left AChA with early drainage into the left basal vein of Rosenthal and vein of Galen (arrowheads). Case 3. E: Right ICA lateral projection of postoperative images demonstrating no residual filling of a right parietal AVM after embolization and microsurgical resection. F: Follow-up angiogram shows recurrent nidus (arrow) in the right parietal lobe with early superficial venous drainage (arrowhead) into the superior sagittal sinus.

Lang et al. presented a cohort in which patients who underwent postoperative DSA at 1 year demonstrated a recurrence rate of zero, while 4 patients who had a postoperative study at an earlier time point presented within 5 years with recurrence. ${ }^{16}$ This finding lends support to the concept that delayed postoperative DSA is needed to monitor for recurrent AVM in children. However, it should be noted that long-term follow-up in our series identified 1 patient with a recurrence 6 years after complete resection with a previously negative surveillance DSA at 4 years (Case 3), as illustrated in Fig. 2. Others have reported recurrence occurring as late as 16 years after initial treat- 
TABLE 4. Characteristics of 3 patients with recurrent AVMs*

\begin{tabular}{ccccc}
\hline $\begin{array}{c}\text { Case } \\
\text { No. }\end{array}$ & $\begin{array}{c}\text { Age } \\
\text { (yrs) }\end{array}$ & $\begin{array}{c}\text { Presentation at } \\
\text { Recurrence }\end{array}$ & $\begin{array}{c}\text { Time to } \\
\text { Recurrence } \\
\text { (yrs) }\end{array}$ & $\begin{array}{c}\text { Spetzler-Martin Grade } \\
\text { (size/eloquence/ } \\
\text { drainage) }\end{array}$ \\
\hline 1 & 6 & Asymptomatic & 1.6 & II (I////0) \\
\hline 2 & 14 & Hemorrhage & 2.8 & IV (II///I) \\
\hline 3 & 8 & Asymptomatic & 6.0 & II (I/O/I) \\
\hline
\end{tabular}

* All 3 patients underwent DSA to diagnose recurrence.

ment. ${ }^{29}$ We affirm that delayed DSA is needed, and contend that repeated studies are essential to diagnose recurrent AVM before catastrophic hemorrhage.

Our findings confirm that long-term follow-up in children is a requirement after AVM treatment. $1,6,20$ We find, consistent with previous reports, that MRI/MRA as performed in this cohort was unhelpful for the detection of subtle recurrence. ${ }^{1}$ Furthermore, subjecting young children to MRI is often not a trivial matter, frequently requiring conscious sedation or general anesthesia to achieve adequate imaging. In our series, 1 patient was found to have a recurrence on DSA after negative MRI and MRA, while another presented with hemorrhage from a recurrent AVM after negative MRI and MRA. The third patient was found to have a recurrent lesion on DSA after an earlier negative DSA. With this knowledge, and the fact that more than one-third of patients were followed with MRI and/or MRA alone in the first 3 years of follow-up and some indefinitely, we postulate that recurrence rates may be even higher than we observed in our cohort.

While our data did not support the value of Gd-enhanced MRI or 3D time of flight MRA in surveillance of fully treated AVMs in children, we must acknowledge that the combination of sequences used over the 14 years of this study does not represent the most sensitive MRI available today. New techniques employing 3-T magnets and the latest 3D contrast-enhanced time-resolved MRA, also known as 4D MRA, are emerging for imaging AVMs. However, the most recent evidence on the subject still demonstrates that DSA is substantially more sensitive in surveillance and detection, although these new modalities do have a role in grading and treatment planning for known lesions. ${ }^{21,25}$ As we observe in Fig. 1, AVM recurrence in our cohort was at times a very subtle finding, reinforcing the idea that noninvasive modalities for follow-up that are currently available may not be sufficient to detect it.

The appropriate follow-up imaging protocol in children with treated AVMs has not been established, although most recommend DSA as a component of the follow-up paradigm. $.1,9,16$ Concern regarding the safety of radiation exposure in children has caused a shift away from studies involving the use of ionizing radiation, such as angiography and $\mathrm{CT}^{7,22}$ Recent estimates have placed the mean radiation dose to the skin from a single diagnostic angiogram in a child under the age of 21 at $239.4 \mathrm{mGy}$, while the dose absorbed by the brain is lower. Infants experience a slightly higher dose due to skull thickness, but the total is still less than $1 \mathrm{~Gy} .{ }^{22}$ White et al. demonstrated that in children undergoing surveillance for shunt malfunction, the mean brain dose from a mean of $16.3 \mathrm{CT}$ scans in

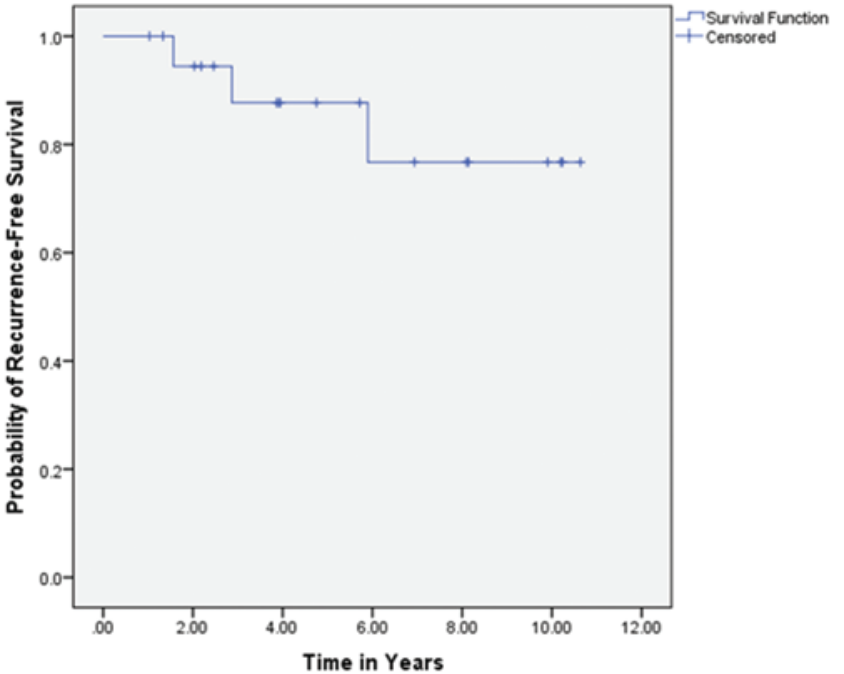

FIG. 2. Kaplan-Meier curve demonstrating recurrence-free survival in the cohort of 20 patients with a completely obliterated AVM nidus after treatment and a minimum of 1 -year follow-up. Figure is available in color online only.

the first year of life was $321 \mathrm{mGy}$, with no increase in the risk of malignancy over the 10-21 years of follow-up. ${ }^{30}$ Consistent with this conclusion, the largest study of population-based risk of malignancy after radiation exposure by dose demonstrated no significant increase in the relative risk of malignancy after exposure to 0.01-9.99 Gy. ${ }^{27}$ Additionally, neurological and nonneurological risks of DSA in the infant population were recently reviewed at our institution with no increase in morbidity in this population. ${ }^{11}$ All of this accumulating evidence suggests that the additional risk of favoring DSA over MRI/MRA in a follow-up protocol after AVM treatment is minimal, and unlikely to surpass the risk of hemorrhage from an undetected recurrence.

We propose an approach in which each patient is evaluated with an intraoperative or early postoperative angiogram to confirm complete resection, followed by a repeat angiographic study at 1 year to confirm the absence of a nidus. After that time, follow-up DSA would be performed at 3 years, which would coincide with the median time to recurrence in our study. Subsequent DSA would be performed again at 5 and 10 years and every 5 years subsequently. The combination of our experience with that of other investigators suggests that this strategy would capture most pediatric recurrences without subjecting children to annual or more frequent invasive vascular studies utilizing ionizing radiation and general anesthesia.,16 As one of our recurrences occurred after a negative DSA, it is also possible that DSA will incompletely diagnose recurrence. Based on our findings, however, the addition of MRI/MRA to the follow-up paradigm is unlikely to increase the sensitivity of diagnosis or prevent symptomatic recurrence between imaging intervals. While a noninvasive study that does not employ ionizing radiation is an attractive alternative to parents and providers, our data call into question the role of MRI/MRA in surveillance after treatment of pediatric AVM. 
This study is limited by a small sample size with few recurrences, consistent with the low overall incidence of AVM hemorrhage in the general pediatric population. As with any retrospective analysis, we are limited by potential bias of treating providers and our data collection. Further, our sample contains few patients with CTA follow-up only, leaving room for further study regarding the validity of CTA as a substitute for DSA in our proposed treatment paradigm. As the quality and sensitivity of CTA or 4D enhanced MRA-generated images improves, one may consider replacement of DSA at interval time points, but this approach will require additional validation.

\section{Conclusions}

Our experience supports the fact that conventional angiography is currently a more sensitive modality for detecting recurrent AVMs than MRI/MRA. Given the apparent lack of benefit attained from MRI/MRA accompanied by the challenges of achieving high-quality results in young children, we conclude that there is little added value to combining MRA with DSA in a postoperative follow-up paradigm. We suggest a surveillance strategy that utilizes DSA at defined intervals so as to maximize the sensitivity of follow-up studies while sparing the child exposure to excessive ionizing radiation, anesthesia, and the risks of conventional angiography.

\section{References}

1. Ali MJ, Bendok BR, Rosenblatt S, Rose JE, Getch CC, Batjer $\mathrm{HH}$ : Recurrence of pediatric cerebral arteriovenous malformations after angiographically documented resection. Pediatr Neurosurg 39:32-38, 2003

2. Andaluz N, Myseros JS, Sathi S, Crone KR, Tew JM Jr: Recurrence of cerebral arteriovenous malformations in children: report of two cases and review of the literature. Surg Neurol 62:324-331, 2004

3. Bendok BR, El Tecle NE, El Ahmadieh TY, Koht A, Gallagher TA, Carroll TJ, et al: Advances and innovations in brain arteriovenous malformation surgery. Neurosurgery 74 (Suppl 1):S60-S73, 2014

4. Beslow LA, Licht DJ, Smith SE, Storm PB, Heuer GG, Zimmerman RA, et al: Predictors of outcome in childhood intracerebral hemorrhage: a prospective consecutive cohort study. Stroke 41:313-318, 2010

5. Blauwblomme T, Bourgeois M, Meyer P, Puget S, Di Rocco F, Boddaert N, et al: Long-term outcome of 106 consecutive pediatric ruptured brain arteriovenous malformations after combined treatment. Stroke 45:1664-1671, 2014

6. Bristol RE, Albuquerque FC, Spetzler RF, Rekate HL, McDougall CG, Zabramski JM: Surgical management of arteriovenous malformations in children. J Neurosurg 105 (2 Suppl):88-93, 2006

7. Chen JX, Kachniarz B, Gilani S, Shin JJ: Risk of malignancy associated with head and neck CT in children: a systematic review. Otolaryngol Head Neck Surg 151:554-566, 2014

8. Di Rocco C, Tamburrini G, Rollo M: Cerebral arteriovenous malformations in children. Acta Neurochir (Wien) 142:145158,2000

9. Gaballah M, Storm PB, Rabinowitz D, Ichord RN, Hurst RW, Krishnamurthy G, et al: Intraoperative cerebral angiography in arteriovenous malformation resection in children: a single institutional experience. J Neurosurg Pediatr 13:222-228, 2014

10. Hladky JP, Lejeune JP, Blond S, Pruvo JP, Dhellemmes P:
Cerebral arteriovenous malformations in children: report on 62 cases. Childs Nerv Syst 10:328-333, 1994

11. Hoffman CE, Santillan A, Rotman L, Gobin YP, Souweidane MM: Complications of cerebral angiography in children younger than 3 years of age. J Neurosurg Pediatr 13:414419,2014

12. Humphreys RP, Hoffman HJ, Drake JM, Rutka JT: Choices in the 1990s for the management of pediatric cerebral arteriovenous malformations. Pediatr Neurosurg 25:277-285, 1996

13. Kader A, Goodrich JT, Sonstein WJ, Stein BM, Carmel PW, Michelsen WJ: Recurrent cerebral arteriovenous malformations after negative postoperative angiograms. J Neurosurg 85:14-18, 1996

14. Klimo P Jr, Rao G, Brockmeyer D: Pediatric arteriovenous malformations: a 15-year experience with an emphasis on residual and recurrent lesions. Childs Nerv Syst 23:31-37, 2007

15. Kondziolka D, Humphreys RP, Hoffman HJ, Hendrick EB, Drake JM: Arteriovenous malformations of the brain in children: a forty year experience. Can J Neurol Sci 19:40-45, 1992

16. Lang SS, Beslow LA, Bailey RL, Vossough A, Ekstrom J, Heuer GG, et al: Follow-up imaging to detect recurrence of surgically treated pediatric arteriovenous malformations. J Neurosurg Pediatr 9:497-504, 2012

17. Millar C, Bissonnette B, Humphreys RP: Cerebral arteriovenous malformations in children. Can J Anaesth 41:321-331, 1994

18. Morgan MK, Patel NJ, Simons M, Ritson EA, Heller GZ: Influence of the combination of patient age and deep venous drainage on brain arteriovenous malformation recurrence after surgery. J Neurosurg 117:934-941, 2012

19. Mullan S, Mojtahedi S, Johnson DL, Macdonald RL: Embryological basis of some aspects of cerebral vascular fistulas and malformations. J Neurosurg 85:1-8, 1996

20. Niazi TN, Klimo P Jr, Anderson RC, Raffel C: Diagnosis and management of arteriovenous malformations in children. Neurosurg Clin N Am 21:443-456, 2010

21. Oleaga L, Dalal SS, Weigele JB, Hurst RW, Lee J, Voorhees A, et al: The role of time-resolved 3D contrast-enhanced MR angiography in the assessment and grading of cerebral arteriovenous malformations. Eur J Radiol 74:e117-e121, 2010

22. Orbach DB, Stamoulis C, Strauss KJ, Manchester J, Smith ER, Scott RM, et al: Neurointerventions in children: radiation exposure and its import. AJNR Am J Neuroradiol 35:650-656, 2014

23. Park YS, Kwon JT: Recurrent cerebral arteriovenous malformation in a child: case report and review of the literature. $\mathbf{J}$ Korean Neurosurg Soc 45:401-404, 2009

24. Rubin BA, Brunswick A, Riina H, Kondziolka D: Advances in radiosurgery for arteriovenous malformations of the brain. Neurosurgery 74 (Suppl 1):S50-S59, 2014

25. Soize S, Bouquigny F, Kadziolka K, Portefaix C, Pierot L: Value of 4D MR angiography at 3T compared with DSA for the follow-up of treated brain arteriovenous malformation. AJNR Am J Neuroradiol 35:1903-1909, 2014

26. Takagi Y, Kikuta K, Nozaki K, Hashimoto N: Early regrowth of juvenile cerebral arteriovenous malformations: report of 3 cases and immunohistochemical analysis. World Neurosurg 73:100-107, 2010

27. Taylor AJ, Little MP, Winter DL, Sugden E, Ellison DW, Stiller CA, et al: Population-based risks of CNS tumors in survivors of childhood cancer: the British Childhood Cancer Survivor Study. J Clin Oncol 28:5287-5293, 2010

28. Vanlandingham M, Fox B, Hoit D, Elijovich L, Arthur AS: Endovascular treatment of intracranial dural arteriovenous fistulas. Neurosurgery 74 (Suppl 1):S42-S49, 2014

29. Weil AG, Li S, Zhao JZ: Recurrence of a cerebral arteriovenous malformation following complete surgical resection: 
A case report and review of the literature. Surg Neurol Int 2:175, 2011

30. White IK, Shaikh KA, Moore RJ, Bullis CL, Sami MT, Gianaris TJ, et al: Risk of radiation-induced malignancies from CT scanning in children who underwent shunt treatment before 6 years of age: a retrospective cohort study with a minimum 10-year follow-up. J Neurosurg Pediatr 13:514-519, 2014

31. Zhao J, Wang S, Li J, Qi W, Sui D, Zhao Y: Clinical characteristics and surgical results of patients with cerebral arteriovenous malformations. Surg Neurol 63:156-161, 2005

32. Zheng T, Wang QJ, Liu YQ, Cui XB, Gao YY, Lai LF, et al: Clinical features and endovascular treatment of intracranial arteriovenous malformations in pediatric patients. Childs Nerv Syst 30:647-653, 2014

\section{Disclosure}

Dr. Souweidane serves as a consultant to Aesculap.

\section{Author Contributions}

Conception and design: Morgenstern, Hoffman, Kocharian, Stieg, Souweidane. Acquisition of data: Morgenstern, Kocharian, Singh. Analysis and interpretation of data: Morgenstern, Hoffman, Kocharian, Singh, Souweidane. Drafting the article: Morgenstern, Hoffman, Singh. Critically revising the article: Morgenstern, Hoffman. Reviewed submitted version of manuscript: all authors. Approved the final version of the manuscript on behalf of all authors: Morgenstern. Statistical analysis: Morgenstern, Kocharian. Administrative/technical/material support: Kocharian. Study supervision: Hoffman, Stieg, Souweidane.

\section{Correspondence}

Peter Morgenstern, Department of Neurological Surgery, NewYork-Presbyterian Hospital, Weill Cornell Medical Center, 525 E. 68th St., Box 99, New York, NY 10065. email: pfmorgenstern @ gmail.com. 